\title{
Comparison of popliteal artery aneurysm outcomes after open repair and endovascular repair: reducing post-operative type II endoleak and sac enlargement
}

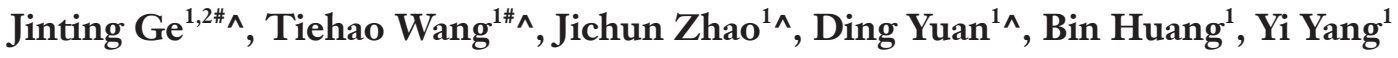 \\ ${ }^{1}$ Department of Vascular Surgery, West China Hospital, Sichuan University, Chengdu, China; ${ }^{2}$ West China School of Medicine, Sichuan University, \\ Chengdu, China \\ Contributions: (I) Conception and design: J Ge, J Zhao; (II) Administrative support: T Wang, J Zhao, B Huang; (III) Provision of study materials or \\ patients: J Ge, T Wang, J Zhao; (IV) Collection and assembly of data: J Ge, T Wang, Y Yang; (V) Data analysis and interpretation: J Ge, T Wang, B \\ Huang, D Yuan, Y Yang; (VI) Manuscript writing: All authors; (VII) Final approval of manuscript: All authors. \\ \#These authors contributed equally to this work. \\ Correspondence to: Jichun Zhao, MD, PhD. Department of Vascular Surgery, West China Hospital, Sichuan University, 37 Guo Xue Alley, Chengdu \\ 610041, China. Email: zhaojichundoc@163.com; Bin Huang, MD. Department of Vascular Surgery, West China Hospital, Sichuan University, 37 \\ Guo Xue Alley, Chengdu 610041, China. Email: dochuangbin@163.com.
}

Background: This cohort study aimed to evaluate the short- and long-term outcomes of open repair (OR) and endovascular repair (ER) and identify the most suitable graft and approach for treating popliteal artery aneurysm (PAA) patients.

Methods: The data of PAA patients from January 2000 to August 2020 were retrospectively collected and analyzed. The primary endpoints were 30-day mortality, peri-operative complication morbidity, postoperative type II endoleak, and sac- enlargement. The secondary endpoints were primary graft patency, secondary graft patency, the reintervention rate, and overall mortality.

Results: A total of 213 PAAs of 186 patients were surgically repaired. The ER group had a significantly lower mean length of hospital stay, blood loss volume, and aneurysm diameter than the OR group. There were no significant differences between the ER and OR groups in terms of average surgery time, 30-day mortality, peri-operative complication morbidity, post-operative type II endoleak, sac enlargement, primary and secondary graft patency, reintervention rate, and overall mortality. However, in the sub-group analysis, autologous vein grafts had significantly higher primary and secondary graft patency rates than expanded polytetrafluoroethylene (ePTFE) vascular grafts and stent grafts. Type II endoleaks and post-operative sac enlargements were less likely to occur in OR patients when the posterior approach was adopted.

Conclusions: We failed to establish any difference in superiority between OR or ER. However, we found that autologous vein grafts have better graft patency and the posterior approach is less likely to lead to type II endoleaks and sac enlargements after the initial PAA surgical procedure. However, more high-quality, largescale randomized controlled trials need to be conducted.

Keywords: Popliteal artery aneurysm (PAA); open repair (OR); endovascular repair (ER); procedure approach; type II endoleak

Submitted Sep 26, 2021. Accepted for publication Nov 12, 2021.

doi: $10.21037 /$ atm-21-5631

View this article at: https://dx.doi.org/10.21037/atm-21-5631

$\wedge$ ORCID: Jinting Ge, 0000-0003-0952-5850; Tiehao Wang, 0000-0002-8901-9765; Jichun Zhao, 0000-0002-1799-9514; Ding Yuan, 0000-0001$8066-8155$. 


\section{Introduction}

Popliteal artery aneurysms (PAAs) are rare but account for $70-80 \%$ of peripheral artery aneurysms, and have an overall incidence of $0.01-0.1 \%(1,2)$. The main causes of PAA include atherosclerosis, trauma, infection, auto-immune diseases, etc. More than half of PAA cases are asymptomatic, but PAAs can cause various complications, including acute or chronic lower limb ischemia, rupture, and major amputation. The 5-year complication rate is reported to be $60-75 \%(3,4)$. Additionally, acute lower limb ischemia leads to major amputation in $30-40 \%$ of PAA patients $(4,5)$.

Since John Hunter first introduced the surgical repair of PAAs in 1795 (6), open repair (OR) with endoaneurysmorrhaphy and venous bypass has been the "gold standard" surgical treatment option for PAA patients. OR produces excellent results, including good graft patency and a low limb loss rate. Despite OR has definitive outcomes, it still has several disadvantages. For example, OR requires general anesthesia and it needs several hours to complete the surgery, which could bring burden to patients' respiratory and circulation system. As technology advances, the use of endovascular repair (ER) in the treatment of PAA has emerged as a potential alternative to OR. ER is more mini-invasive when compared with OR, it only requires local anesthesia upon most occasions and needs less operation time than OR. However, ER's long-term follow up results remains unclear. To date, no consensus has been reached among vascular surgeons as to the best treatment for PAA, and several studies have failed to demonstrate a difference in superiority between OR and ER (7-17). Post-operative type II endoleak is a rare but highly complex complication after PAA surgery and is extremely difficult to treat.

Our study used data from a 20-year period to evaluate the short- and long-term outcomes of OR and ER. Furthermore, in view of the choice of conduit and surgery approach may affect the prognosis of PAA patients, our research innovatively evaluated which conduit or approach is the most suitable and can reduce the incidence of type II endoleak and sac enlargement in PAA patients. We present the following article in accordance with the STROBE reporting checklist (available at https://dx.doi.org/10.21037/ atm-21-5631).

\section{Methods}

\section{Study population}

The data of consecutive patients diagnosed with PAA and treated at West China Hospital, Chengdu, Sichuan, China from January 2000 to August 2020 were retrospectively collected from a database for review and analysis. The data collected included demographic, pre-operative comorbidity, clinical assessment, and operative data, and early and late follow-up results. Patients were divided into the OR group or ER group based on the intervention type. Patients in the OR group were sub-divided into further groups based on the procedure used.

\section{Diagnostic and intervention criteria for $P A A$}

A diagnosis of PAA relied on a combination of clinical symptoms (i.e., acute ischemia, popliteal pulsatile mass, claudication/rest pain, neurological sign, or rupture), a physical examination, and auxiliary examinations [e.g., duplex ultrasonography, computed tomography angiography (CTA), or magnetic resonance angiography (MRA)]. The diagnosis criteria for PAA is a focal dilation of the popliteal artery more than $50 \%$ of the normal diameter (see Figure $1 A, 1 B$ ), while the type II endoleak was defined as persistent retrograde blood flow to the aneurysm sac from the branch artery (10).

For asymptomatic PAA patients, the indication that required intervention was a sac diameter $>20 \mathrm{~mm}$. For symptomatic PAA patients, the indication was not related to the aneurysm diameter. Either an autologous vein or an expanded polytetrafluoroethylene (ePTFE) vascular graft (Gore-Tex, W. L. Gore \& Associates, Flagstaff, AZ, USA) was used in the OR procedures. The self-expanding Viabahn stent graft (W. L.Gore \& Associates, Flagstaff, AZ, USA) was used in the ER procedures.

\section{Peri-operative and follow-up management}

Before being discharged from the hospital, all patients received CTA, MRA, or duplex ultrasonography to evaluate the treatment outcomes (see Figure 1C). The patients were prescribed double antiplatelet medications (aspirin $100 \mathrm{mg}$ and clopidogrel $75 \mathrm{mg}$ ) immediately after surgery. Certain patients, such as those with PAA accompanied by arterial thrombosis or embolism, were prescribed antiplatelet and anticoagulation medications $(2.5 \mathrm{mg}$ of rivaroxaban twice a day). The duration of oral medications varied from 3 months to a lifetime, depending on the surgeons' preference, the patients' clinical symptoms and the examination results. The patients were advised to return to the clinic at 1,3 , and 6 months, and annually thereafter for further follow-up 

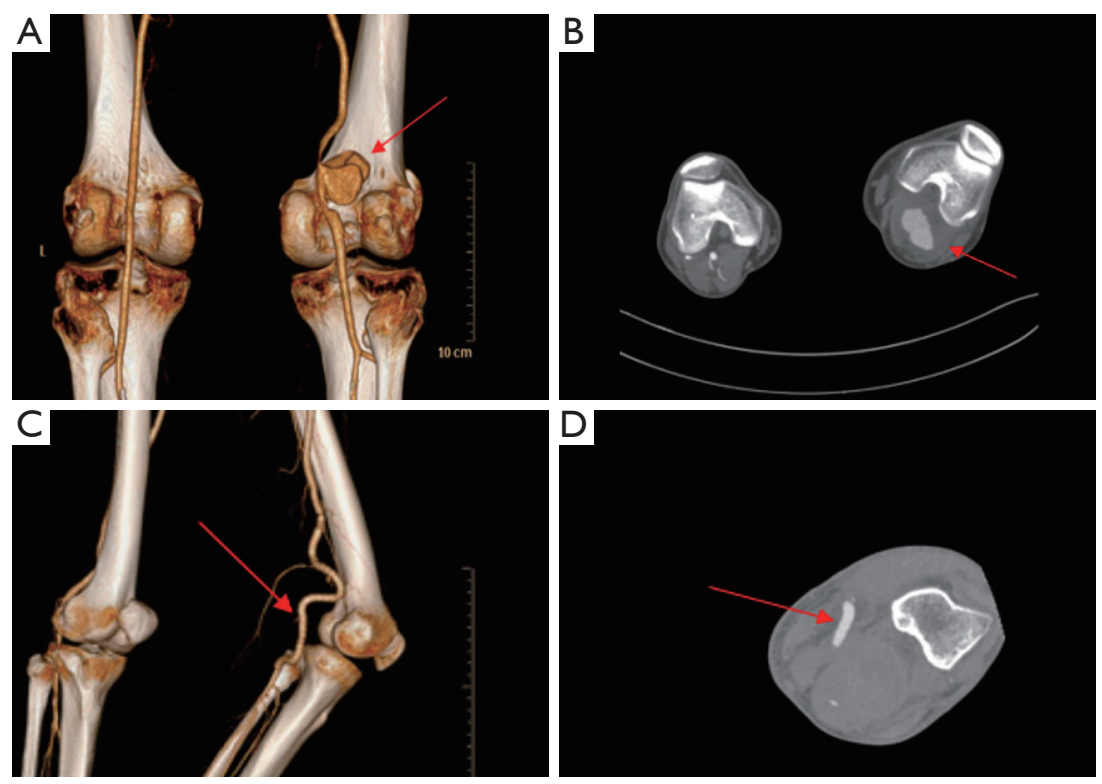

Figure 1 Representative pre- and post-operative images of PAA. (A) A pre-operative three-dimensional reconstruction CTA image of a PAA in right lower limb (red arrow indicates PAA); (B) a pre-operative cross sectional CTA image of PAA in left lower limb, which indicates an enlarged aneurysm and mural thrombosis in aneurysm sac (red arrow indicates PAA and mural thrombosis in the sac); (C) a postoperative three-dimensional reconstruction CTA image of a PAA patient after receiving vascular graft bypass surgery for 1 month and CTA proves the patency of vascular graft (red arrow indicates the three-dimensional CTA image of vascular graft 1 month after operation); (D) a post-operative cross sectional CTA image of a PAA patient after bypass surgery for 6 month, CTA indicates that the vascular graft was unobstructed and complete thrombosis in aneurysm sac (red arrow indicates the vascular graft 6 months after operation). CTA, computed tomography angiography; PAA, popliteal artery aneurysm.

examinations. Physical examinations for arterial pulse and auxiliary examinations (duplex ultrasonography or CTA) were conducted to evaluate vascular patency at the followup clinical examinations (Figure 1D).

\section{Primary and secondary endpoints}

The primary endpoints of our study were 30-day mortality, peri-operative complication morbidity, post-operative type II endoleak, and sac enlargement. The secondary endpoints were primary graft patency, secondary graft patency, the reintervention rate, and overall mortality.

\section{Statistical analysis}

The student $t$-test was used to analyze differences between the means. For the analysis of the categorical variables, Pearson's chi-square test or Fisher's exact test was used. The post-operative sac-enlargement rate, freedom from reintervention, primary graft patency, and overall mortality are presented as Kaplan-Meier curves, and the differences were analyzed by log-rank tests. A P value $<0.05$ was used to define statistical significance. All the statistical data were processed and analyzed by SPSS 21.0 (IBM SPSS, Armonk, NY, USA).

\section{Ethics approval and informed consent}

This study was approved by the Ethics Committee of West China Hospital, Sichuan University (No. 2021-150). We confirm that the research reported in this paper complied with the Helsinki Declaration (as revised in 2013). All patients and/or their authorized trustee were aware that their personal details/images/videos would be collected and analyzed for scientific purposes and might be published. All participants provided written informed consent for the use of their data and the publication of this research.

\section{Results}

From January 2000 to August 2020, 191 patients were diagnosed with PAA at our hospital. The patients had a 
Table 1 Baseline information, pre-operative comorbidities, and clinical symptoms of PAA patients

\begin{tabular}{|c|c|c|c|}
\hline Variables & OR group $(n=151)$ & ER group $(n=35)$ & $\mathrm{P}$ \\
\hline Male gender & $139[92]$ & $30[86]$ & 0.32 \\
\hline \multicolumn{4}{|l|}{ Pre-operative comorbidity } \\
\hline Hypertension & 59 [39] & $16[46]$ & 0.57 \\
\hline Diabetes mellitus & 37 [25] & $12[34]$ & 0.29 \\
\hline Chronic obstructive pulmonary disease & 19 [13] & $6[17]$ & 0.58 \\
\hline Chronic kidney disease & $12[8]$ & $2[6]$ & 1.0 \\
\hline Hyperlipemia & $41[27]$ & $11[31]$ & 0.68 \\
\hline Asymptomatic & $43[28]$ & $19[54]$ & $<0.01$ \\
\hline Claudication/rest pain & $36[24]$ & $5[14]$ & 0.26 \\
\hline Acute ischemia & $26[17]$ & 3 [9] & 0.30 \\
\hline Popliteal pulsatile mass & $25[17]$ & $6[17]$ & 1.0 \\
\hline Neurological sign & $7[5]$ & $2[6]$ & 0.67 \\
\hline Rupture & 3 [2] & $1[3]$ & 0.56 \\
\hline
\end{tabular}

Unless otherwise stated, data are presented as the $n[\%]$ or median \pm standard error. PAA, popliteal artery aneurysm; OR, open repair; ER, endovascular repair.

mean age of $63.6 \pm 16.0$ years, and $17(9 \%)$ were female. Five (3\%) patients whose aneurysm diameters did not meet the criteria for intervention received medication therapy and adhered to a strict follow-up procedure. Surgery repair was performed on 213 PAAs in 186 patients (151 OR and 35 ER patients). Among the OR patients, an autologous vein was used in 100 patients, and an ePTFE vascular graft was used in 51 patients. Among the 186 patients who underwent surgery repair, 77 (41\%) were asymptomatic. The most common symptom was claudication or rest pain, which was observed in $41(22 \%)$ patients, followed by a popliteal pulsatile mass in 31 (17\%) patients, acute ischemia in 29 $(16 \%)$ patients, a neurological sign in $9(5 \%)$ patients, and rupture in $4(2 \%)$ patients. In relation to pre-operative comorbidities, hypertension was observed in 75 (40\%) patients. Further information about patients' demographics, clinical symptoms, and pre-operative comorbidities is presented in Table 1.

Overall, 57 (31\%) patients underwent catheter-directed thrombolysis (CDT) and/or embolectomy before or during the PAA repair procedure. The average duration of CDT was $38.1 \pm 15.6 \mathrm{~h}$. The ER group had a significantly lower mean length of hospital stay $(6.4 \pm 2.4 v s .11 .7 \pm 7.0$ days; $\mathrm{P}<0.01)$, blood loss volume $(66.1 \pm 39.0$ vs. $186.4 \pm 135.9 \mathrm{~mL}$; $\mathrm{P}<0.01)$, and aneurysm diameter $(24.9 \pm 3.5$ vs. $32.1 \pm 7.3 \mathrm{~mm}$; $\mathrm{P}<0.01)$. However, the ER and OR groups showed no significant difference in the average surgery time $(2.56 \pm 0.91$ vs. $2.53 \pm 0.79 \mathrm{~h} ; \mathrm{P}=0.92)$. Patients who underwent the ER procedure were more likely to be asymptomatic (19/35 vs. $43 / 151 ; \mathrm{P}=0.005)$ than those who underwent the OR procedure. Table 2 provides further anatomical and procedural information.

\section{Early outcomes and late follow-up results}

\section{0-day mortality and peri-operative complication morbidity}

During the peri-operative period, only 1 death occurred in the OR group, and no deaths occurred in the ER group $[\mathrm{P}=$ not significant $(\mathrm{NS})]$. The peri-operative mortality rate 
Table 2 Anatomical characteristics and operation/hospital information of PAA patients

\begin{tabular}{|c|c|c|c|}
\hline Variables & $\begin{array}{l}\text { OR group } \\
(n=151)\end{array}$ & $\begin{array}{l}\text { ER group } \\
\qquad(n=35)\end{array}$ & $\mathrm{P}$ \\
\hline \multicolumn{4}{|l|}{ Anatomical characteristic } \\
\hline Aneurysm diameter (mm) & $32.1 \pm 7.3$ & $24.9 \pm 3.5$ & $<0.01$ \\
\hline Bilateral PAA & $23[15]$ & $4[11]$ & 0.79 \\
\hline Run-off score $<2$ & 46 [30] & 10 [29] & 1.0 \\
\hline \multicolumn{4}{|l|}{ Operation information } \\
\hline Blood loss volume (mL) & $186.4 \pm 135.9$ & $66.1 \pm 39.0$ & $<0.01$ \\
\hline $\begin{array}{l}\text { Average surgery time } \\
\text { (hours) }\end{array}$ & $2.53 \pm 0.79$ & $2.56 \pm 0.91$ & 0.92 \\
\hline $\begin{array}{l}\text { Length of hospital stay } \\
\text { (days) }\end{array}$ & $11.7 \pm 7.0$ & $6.4 \pm 2.4$ & $<0.01$ \\
\hline \multicolumn{4}{|l|}{ Other aneurysms } \\
\hline Abdominal aortic & $17[11]$ & 3 [9] & 0.77 \\
\hline Iliac & $9[6]$ & $1[3]$ & 0.69 \\
\hline Renal & $2[1]$ & - & - \\
\hline Splenic & $1[0.7]$ & - & - \\
\hline Femoral & $1[0.7]$ & - & - \\
\hline
\end{tabular}

Unless otherwise stated, data are presented as the $n$ [\%] or median \pm standard error. PAA, popliteal artery aneurysm; OR, open repair; ER, endovascular repair.

was $0.5 \%$. The deceased patient had a surgical history of endovascular aneurysm repair before undergoing the PAA bypass procedure, and suffered a fatal cerebral hemorrhage in the intensive care unit.

Peri-operative complications occurred in 7 OR patients and 2 ER patients $(\mathrm{P}=0.67)$. The peri-operative complication morbidity was $4.8 \%$. The most common complication was acute thrombosis, which was observed in 2 OR patients and 1 ER patient. All of these patients underwent emergency embolectomy, and 1 patient underwent a further vascular graft bypass surgery; however, no major amputation procedure was performed. Other complications included incisional wound infections ( 2 patients in the OR group), pseudoaneurysms ( 1 patient in the OR group and 1 patient in the ER group), major bleeding (1 patient in the OR group), and cerebral hemorrhage (1 patient in the OR group as mentioned above). Emergency procedures were performed to excise the pseudoaneurysms, detect major bleeding, and repair damaged vessels. For patients with wound infections, abscess incision and irrigation were
Table 3 Peri-operative mortality and complication morbidity information of PAA patients

\begin{tabular}{lccc}
\hline Variables & $\begin{array}{c}\text { OR group } \\
(\mathrm{n}=151)\end{array}$ & $\begin{array}{c}\text { ER group } \\
(\mathrm{n}=35)\end{array}$ & $\mathrm{P}$ \\
\hline Peri-operative mortality & $1[0.7]$ & - & - \\
30-day mortality & $2[1]$ & $1[3]$ & 0.08 \\
Peri-operative complication morbidity & & - \\
Acute thrombosis & $2[1]$ & - & - \\
Incisional wound infections & $1[0.7]$ & $1[3]$ & 0.16 \\
Pseudoaneurysms & $1[0.7]$ & - & - \\
Major bleeding & $1[0.7]$ & - & - \\
Cerebral hemorrhage & $7[5]$ & $2[6]$ & 0.67 \\
Total & &
\end{tabular}

Unless otherwise stated, data are presented as the $\mathrm{n}$ [\%]. PAA, popliteal artery aneurysm; OR, open repair, ER, endovascular repair.

performed. All patients recovered well after reintervention. Table 3 provides further information about peri-operative mortality and complication morbidity.

\section{The incidence of post-operative type II endoleaks and sac enlargements}

During the follow-up period, 18 patients had a type II endoleak (14 in the OR group and 4 in the ER group). Among these patients, 6 underwent secondary OR (including ligation of retrograde branch artery and sac resection) to manage the endoleak and aneurysm due to an enlarged sac diameter and the presence of clinical symptoms, while the remaining patients adhered to a strict follow-up procedure due to the lack of clinical symptoms. No significant difference in the incidence of type II endoleaks was found between the OR and ER groups $(\mathrm{P}=0.751)$.

In the OR group, a medial approach was adopted for 85 patients, and a posterior approach for 66 patients. The sub-group analysis revealed that patients who underwent the posterior procedure in the OR group had a lower chance of developing type II endoleaks than those who underwent the medial procedure in the OR group or those in the ER group (1/66 vs. $13 / 85$ and $4 / 35 ; \mathrm{P}=0.004$ and 0.048 , respectively).

Sac enlargement was observed in 22 OR patients and 6 ER patients. Of the 22 OR patients, 20 patients underwent the medial procedure, and 2 underwent the 


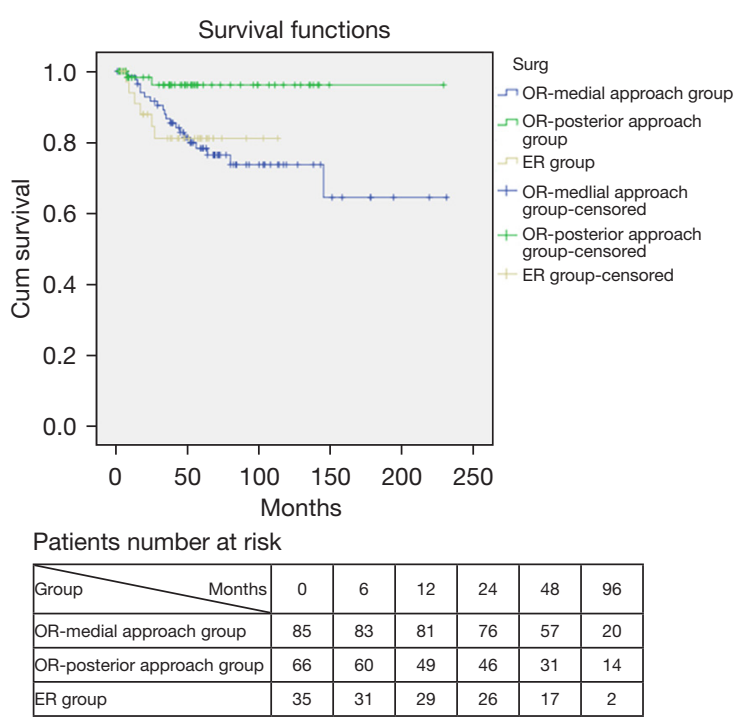

Figure 2 Kaplan-Meier curve of post-operative sac enlargement incidence with numbers of patients at risk.

posterior procedure There was no statistical difference in the incidence of post-operative sac enlargement between the OR and ER groups $(\mathrm{P}=0.470)$; however, the log-rank test revealed that OR patients who underwent the posterior procedure had a lower chance of suffering from postoperative sac enlargement than OR patients who underwent the medial procedure or ER patients $(\mathrm{P}=0.009$ and 0.024 , respectively). The results of the survival analysis are presented in the Kaplan-Meier curve (see Figure 2).

\section{Primary and secondary graft patency}

The average follow-up time was $61.7 \pm 45.5$ months. In total, 36 cases of late thrombosis were documented, including 11 cases in the OR autologous-vein group, 17 cases in the OR ePTFE vascular-graft group, and 8 cases in the ER group. Reintervention procedures were performed for 29 patients with thrombosis, and the other 5 patients were prescribed medication because their ischemia symptoms were mild. However, 4 cases of major amputation were unavoidable. Recurrent thrombosis occurred in 8 patients despite successful reintervention surgeries and led to 3 cases of major amputation. The limb salvage rates at 1,3 , and 5 years were $98.4 \%, 96.8 \%$, and $96.2 \%$, respectively.

The estimated primary graft patency rates in the OR autologous-vein group at 1, 3, and 5 years were $100 \%$, $97.3 \%$, and $95.7 \%$, respectively, after the initial procedure; the corresponding rates in the OR vascular-graft group were $88.9 \%, 86.3 \%$, and $79.2 \%$, respectively, and the ER

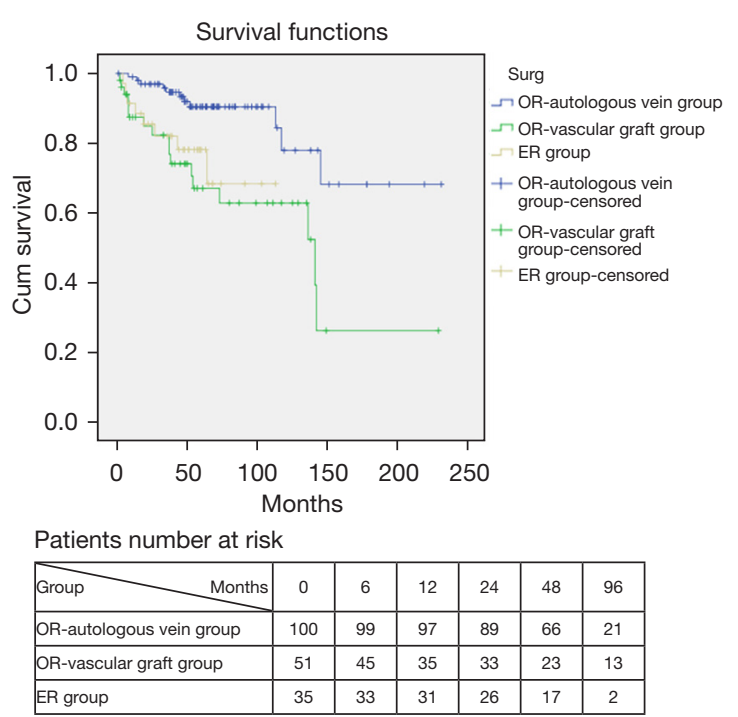

Figure 3 Kaplan-Meier curve of primary graft patency with numbers of patients at risk.

group's primary graft patency rates were $91.4 \%, 88.3 \%$, and $84.1 \%$, respectively. The log-rank test showed that the primary graft patency in the OR autologous-vein group was significantly higher than that in the OR vascular-graft group and the ER group $(\mathrm{P}<0.001$ and $\mathrm{P}=0.011$, respectively; see Figure 3).

Similarly, the estimated secondary graft patency rates were significantly higher in the autologous-vein group (99.0\%, $95.5 \%$, and $93.9 \%$ at 1,3 , and 5 years, respectively) than the vascular-graft group $(94.0 \%, 87.9 \%$, and $79.0 \%$ at 1,3 , and 5 years, respectively) and the ER group (91.4\%, $73.5 \%$, and $68.6 \%$ at 1,3 , and 5 years, respectively) based on the log-rank tests $(\mathrm{P}<0.001$ and $\mathrm{P}=0.009$, respectively). The results are presented in the Kaplan-Meier curve (see Figure 4).

However, when the OR group was compared to the ER group without grouping patients according to the conduits, the OR group's primary graft patency rates at 1,3 , and 5 years were $95.2 \%, 89.0 \%$, and $83.1 \%$, respectively, which were not significantly better than those of the ER group $(\mathrm{P}=0.184)$. The corresponding rates in the ER group were $91.4 \%, 82.0 \%$, and $78.1 \%$, respectively. In addition, the secondary graft patency rates were not statistically different between the OR and ER groups $(\mathrm{P}=0.191)$.

The results of the univariate analysis of the 186 patients who underwent surgical repair showed that the factors affecting patients' graft patency included a poor run-off status, pre-operative severe ischemia symptoms, and an 


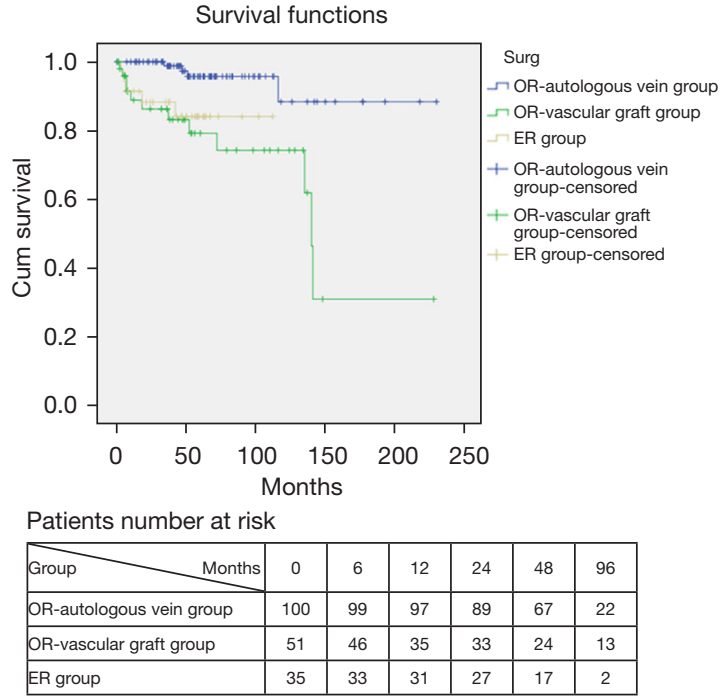

Figure 4 Kaplan-Meier curve of secondary graft patency with numbers of patients at risk.

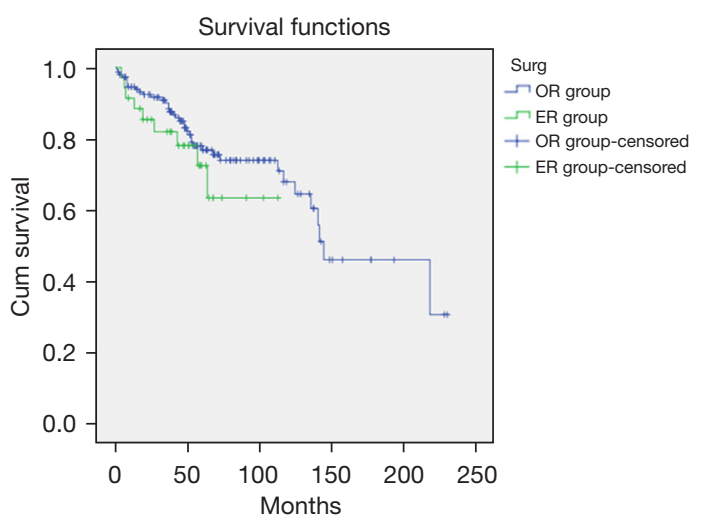

Patients number at risk

\begin{tabular}{|l|c|c|c|c|c|c|}
\hline Group Months & 0 & 6 & 12 & 24 & 48 & 96 \\
\hline OR group & 151 & 144 & 132 & 122 & 87 & 34 \\
\hline ER group & 35 & 32 & 30 & 26 & 17 & 2 \\
\hline
\end{tabular}

Figure 5 Kaplan-Meier curve of freedom of reintervention with numbers of patients at risk.

additional distal vessel procedure.

\section{Freedom from reintervention}

The freedom from reintervention rates in the OR group at 1,3 , and 5 years were $94.6 \%, 88.4 \%$, and $76.9 \%$, respectively. In the ER group, the rates at 1,3 , and 5 years were $91.4 \%, 82.0 \%$, and $72.5 \%$, respectively. The reintervention rates are presented in the Kaplan-Meier curve in Figure 5. The difference in the reintervention rates

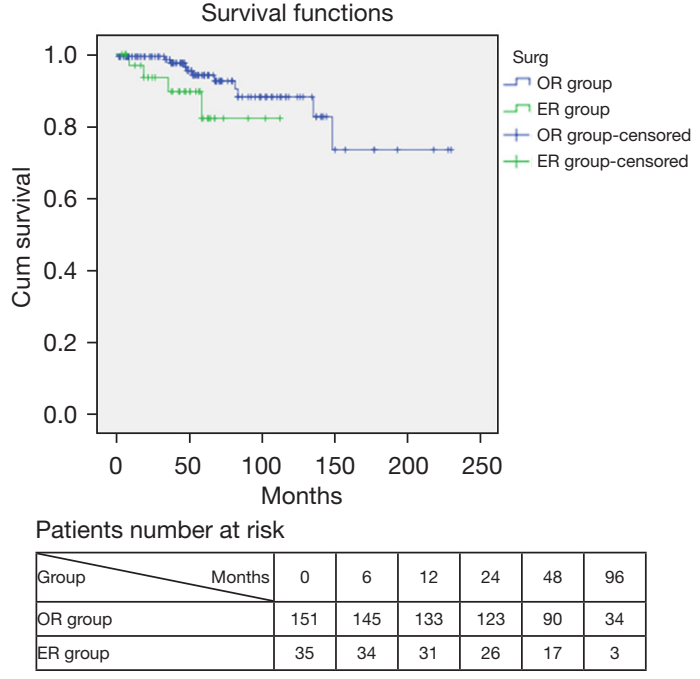

Figure 6 Kaplan-Meier curve of overall mortality with numbers of patients at risk.

was analyzed by a log-rank test, but no difference in the reintervention rates between the OR and ER groups was found $(\mathrm{P}=0.314)$.

\section{Overall mortality}

During the follow-up period, 14 late deaths were recorded (10 in the OR group and 4 in the ER group). Heart failure $(n=3)$ was the most common cause of death; however, other causes of death included acute myocardial infarction $(\mathrm{n}=2)$, chronic kidney dysfunction $(\mathrm{n}=2)$, respiratory failure $(n=2)$, cerebral stroke $(n=1)$, pulmonary embolism $(n=1)$, gastrointestinal bleeding $(\mathrm{n}=1)$, and unknown causes $(n=2)$. The estimated survival rates in the OR group were $99.3 \%$ at 1 year, $98.5 \%$ at 3 years, and $94.2 \%$ at 5 years. The ER group's survival rates at 1,3 , and 5 years were $96.9 \% .89 .6 \%$, and $82.2 \%$, respectively. Figure 6 shows the estimated survival rates in a Kaplan-Meier curve, and a logrank test revealed that there was no statistical difference in the survival rates between the $\mathrm{OR}$ and $\mathrm{ER}$ groups $(\mathrm{P}=0.11)$. The overall estimated survival rates for all 186 patients at 1 , 3 , and 5 years were $98.9 \%, 96.8 \%$, and $92.1 \%$, respectively.

\section{Discussion}

Traditional OR for PAA provides excellent results and is still the "gold standard" in PAA treatment (18-20). However, with the progress in endovascular materials and technology, more and more ER procedures are 
being performed on PAA patients. Several studies on OR and ER have been published in recent years; however (10,14-16,21-24), few have compared the graft patency of different conduits $(14,20)$, which include autologous vein grafts, ePTFE vascular grafts, and stent grafts. In our study, we found that among the different conduits, an autologous vein was the best conduit for PAA patients due to its higher graft patency. Additionally, there was no significant difference in OR and ER showed based on the early and follow-up results. However, the ER group had a lower mean length of hospital stay, blood loss volume, and aneurysm diameter, indicating that ER may be a better choice for patients with a high peri-operative risk and good anatomical characteristics. We also found that PAA patients with complex anatomical characteristics or severe ischemia were more likely to undergo OR procedures in our study.

Today, the traditional medial approach, which includes the ligation of the proximal and distal ends of the popliteal artery and the autologous vein or an ePTFE graft bypass, is the most common open surgery technique for PAA. Surgery that adopts the medial approach is easy; however, it leaves the aneurysm itself and the branch artery untreated, which can cause persistent blood backflow, reperfusion, and sac enlargement. Endoleak after the PAA repair procedure has been reported to have a morbidity rate of $8 \%$, and can lead to aneurysm rupture in rare cases $(12,25,26)$. Tsilimparis et al. also reported that sac enlargement occurred in up to $36 \%$ of patients who underwent the medial procedure (27).

The posterior approach was introduced as an alternative route in OR. This clearly exposes the aneurysm and allows for the definitive exclusion of the aneurysm and branch artery, which reduces the incidence of endoleak and sac enlargement. However, the posterior approach is more complex than the medial approach because the popliteal vein and tibial nerve may be injured during the aneurysm exclusion process. In our study, we found that the posterior technique significantly reduced the incidence of postoperative type II endoleak and sac enlargement.

There are currently no standard protocols for vascular surgeons to follow when handling type II endoleaks. Sac enlargement accompanied by obvious clinical symptoms is an indication for secondary intervention at our institution. Normal surgery for type II endoleaks and sac enlargements includes ligation of retrograde branch artery and sac resection. Interestingly, we recently used fibrin sealant to prevent type II endoleak in a patient with PAA rupture (28). The fibrin sealant was injected directly into the aneurysm sac in our procedure, and no signs of endoleak were observed during the follow-up period, indicating that it may be an alternative choice in PAA surgery.

Mehta et al. suggested that routine post-operative surveillance of the aneurysm sac be performed for all patients who undergo surgical procedures for PAAs (29). Interestingly, our findings revealed that the risk of endoleak and sac enlargement increased with the length of the followup period (see Figure 2). In our study, the incidence of sac enlargement increased from $7.2 \%$ to $16.4 \%$ after followup periods of 24 and 60 months, respectively, indicating that routine post-operative surveillance is necessary for PAA patients.

Acute ischemia resulting from PAA thrombosis is associated with a high limb-threatening risk (30). When acute ischemia occurs, emergency PAA exclusion or resection plus autologous vein bypass is usually performed, which is still the standard procedure for PAA patients. However, a few PAA thrombosis patients also suffer from distal tibial and peroneal artery stenosis or occlusion, which contributes to poor run-off conditions and leads to early graft thrombosis, graft failure, and major amputation. Schwarz et al. introduced pre-operative intra-arterial thrombolysis in 1984 to improve the revascularization and patency of distal vessels (31). Dragas et al. noted that CDT prior to surgery can reduce long-term major adverse limb events and provide overall survival benefits in selected patients with no risk of increasing major or lifethreatening bleeding complications (30). However, it should be noted that a review conducted in 2010 revealed that preoperative thrombolysis did not improve the limb salvage rate (32). Thrombolysis may not be appropriate for severe acute ischemia patients as the revascularization of distal vessels often requires a massive dose of thrombolytics and prolonged time, which may cause irreversible ischemia and necessitate major amputation. Direct embolectomy may be an alternative approach and has an acceptable efficacy in treating acute ischemia patients with PAA.

Similar to previous studies $(12,14)$, we demonstrated that poor run-off status, pre-operative severe ischemia, and the additional distal vessel procedure are reliable factors for predicting the patients' graft patency. An earlier study identified diabetes as a factor influencing graft patency. Notably, the distal vessel condition of patients with diabetes is usually poorer than that of normal patients (12). However, our study did not confirm these findings. For patients with poor distal vessel conditions, we will try to improve outflow with an additional PTA or endarterectomy procedure. We believe that additional procedures can prevent early 
thrombosis; however, Maraglino et al. reported that such procedures had low efficacy in improving long-term patency due to heavily calcified distal vessels (12), which is consistent with the results of our study.

PAA, especially bilateral PAA, is associated with a higher risk of abdominal aortic aneurysm (AAA). A retrospective study published in 2018 reported that patients with bilateral PAA and AAA had a lower risk at of graft occlusion (12), which may be attributed to early diagnosis and treatment. This was not confirmed in the present study; however, we believe that it is necessary to screen PAA patients for AAA. Further, no consensus has been reached as to the best treatment for patients with PAA accompanied by AAA. In our experience, when PAA and AAA both require interventions but the patient is asymptomatic, AAA should be prioritized.

Any PAA treatment policy should consider the patient's general status, clinical presentation, anatomical characteristics, pre-operative comorbidities, and life expectancy. In our daily practice, we have preferred the posterior approach in recent years due to its capacity to reduce post-operative endoleak and sac enlargement. An autologous vein is our first choice of conduit due to the high graft patency and excellent follow-up results. Vascular graft is appliable under the circumstances that great saphenous vein is not considered in the choice of conduits. For example, the great saphenous vein cannot be harvested when patient is diagnosed with venous insufficiency or deep vein thrombosis. Low general status, malignant tumors, an inability to tolerate general anesthesia, and other severe comorbidities are indications for ER in PAA patients. Complications of PAA are various and severe, sometimes limb-threatening. Therefore, strict observation during perioperative period and appropriate follow-up management are essential. As has been stated, posterior approach can reduce the incidence of post-operative type II endoleak and sac enlargement, thus reduce post-operative complications and reintervention. Additionally, physical examination of the dorsal artery and posterior tibial artery during perioperative and follow-up period is also important because it is a non-invasive method to examine whether popliteal artery and bypass graft is stenosis or occluded. Moreover, postoperative double anti-platelet medications, plus statin or anti-coagulation drugs, are extremely important because they are capable of preventing thrombosis and graft occlusion.

The main limitation of our study is that it was a retrospective and non-randomized study with only a single participating center, which may have caused selection bias. Further, the number of patients in the ER group was relatively small. Our study failed to show the superiority of OR or ER; however, its strengths lie in the relatively large number of patients, long follow-up period, and positive findings regarding the conduit and approach choice. We demonstrated that OR with autologous vein bypass remains the "gold standard" for PAA patients and that the posterior approach could help to reduce the incidence of postoperative type II endoleaks and sac enlargements.

\section{Conclusions}

We failed to establish any difference in superiority between OR or ER. Indeed, both treatments can produce excellent early and follow-up results. An autologous vein appears to be a better conduit than a vascular graft and a stent graft due to its higher graft patency. Additionally, the posterior approach in the OR procedure can reduce the incidence of post-operative type II endoleaks and sac enlargements, and thus possibly prevent the need for a secondary intervention. In summary, OR with autologous vein bypass remains the gold standard for PAA patients, and the posterior approach is an excellent option. Currently, only 2 multicenter trials involving PAAs have been published, and there are no specific guidelines for surgeons to follow $(14,17,18)$. Thus, more high-quality, large-scale randomized controlled trials need to be conducted.

\section{Acknowledgments}

Funding: This work was supported by the Sichuan Science and Technology Program and the Sichuan Foundation of Science and Technology [Grant numbers: 2019JDRC0104, 2019YJ066]. The funding bodies played no role in the design of the study, the collection, analysis, and interpretation of the data, and the writing of the manuscript.

\section{Footnote}

Reporting Checklist: The authors have completed the STROBE reporting checklist. Available at https://dx.doi. org/10.21037/atm-21-5631

Data Sharing Statement: Available at https://dx.doi. org/10.21037/atm-21-5631

Conflicts of Interest: All authors have completed the ICMJE 
uniform disclosure form (available at https://dx.doi. org/10.21037/atm-21-5631). The authors have no conflicts of interest to declare.

Ethical Statement: The authors are accountable for all aspects of the work in ensuring that questions related to the accuracy or integrity of any part of the work are appropriately investigated and resolved. This study was approved by the Ethics Committee of West China Hospital, Sichuan University (No. 2021-150). All patients and/or their authorized trustees were aware of that their personal details/images/videos would be collected and analyzed for scientific purposes and might be published. All participants provided written informed consent for the use of their data and the publication of this research. We confirm that the research reported in this paper complied with the Helsinki Declaration (as revised in 2013).

Open Access Statement: This is an Open Access article distributed in accordance with the Creative Commons Attribution-NonCommercial-NoDerivs 4.0 International License (CC BY-NC-ND 4.0), which permits the noncommercial replication and distribution of the article with the strict proviso that no changes or edits are made and the original work is properly cited (including links to both the formal publication through the relevant DOI and the license). See: https://creativecommons.org/licenses/by-nc-nd/4.0/.

\section{References}

1. Lawrence PF, Lorenzo-Rivero S, Lyon JL. The incidence of iliac, femoral, and popliteal artery aneurysms in hospitalized patients. J Vasc Surg 1995;22:409-15; discussion 415-6.

2. Dent TL, Lindenauer SM, Ernst CB, et al. Multiple arteriosclerotic arterial aneurysms. Arch Surg 1972;105:338-44.

3. Whitehouse WM Jr, Wakefield TW, Graham LM, et al. Limb-threatening potential of arteriosclerotic popliteal artery aneurysms. Surgery 1983;93:694-9.

4. Dawson I, Sie R, van Baalen JM, et al. Asymptomatic popliteal aneurysm: elective operation versus conservative follow-up. Br J Surg 1994;81:1504-7.

5. Dawson I, Sie RB, van Bockel JH. Atherosclerotic popliteal aneurysm. Br J Surg 1997;84:293-9.

6. Perry MO. John Hunter--triumph and tragedy. J Vasc Surg 1993;17:7-14.

7. Kropman RH, van Meurs A, Fioole B, et al. Association of sex with long-term outcomes after popliteal artery aneurysm repair. Ann Vasc Surg 2014;28:338-44.

8. Björck M, Beiles B, Menyhei G, et al. Editor's Choice: Contemporary treatment of popliteal artery aneurysm in eight countries: A Report from the Vascunet collaboration of registries. Eur J Vasc Endovasc Surg 2014;47:164-71.

9. Jung E, Jim J, Rubin BG, et al. Long-term outcome of endovascular popliteal artery aneurysm repair. Ann Vasc Surg 2010;24:871-5.

10. Huang Y, Gloviczki P, Oderich GS, et al. Outcomes of endovascular and contemporary open surgical repairs of popliteal artery aneurysm. J Vasc Surg 2014;60:631-8.e2.

11. Berceli SA. Endovascular treatment of popliteal artery aneurysms: Results of a prospective cohort study. Yearbook of Vascular Surgery 2007;225-6.

12. Maraglino C, Canu G, Ambrosi R, et al. Endovascular Treatment of Popliteal Artery Aneurysms: A Word of Caution after Long-Term Follow-up. Ann Vasc Surg 2017;41:62-8.

13. Wrede A, Wiberg F, Acosta S. Increasing the Elective Endovascular to Open Repair Ratio of Popliteal Artery Aneurysm. Vasc Endovascular Surg 2018;52:115-23.

14. Pulli R, Dorigo W, Castelli P, et al. A multicentric experience with open surgical repair and endovascular exclusion of popliteal artery aneurysms. Eur J Vasc Endovasc Surg 2013;45:357-63.

15. Dorigo W, Fargion A, Masciello F, et al. A Matched Case-Control Study on Open and Endovascular Treatment of Popliteal Artery Aneurysms. Scand J Surg 2018;107:236-43.

16. Leake AE, Avgerinos ED, Chaer RA, et al. Contemporary outcomes of open and endovascular popliteal artery aneurysm repair. J Vasc Surg 2016;63:70-6.

17. Aboyans V, Ricco JB, Bartelink MEL, et al. Editor's Choice - 2017 ESC Guidelines on the Diagnosis and Treatment of Peripheral Arterial Diseases, in collaboration with the European Society for Vascular Surgery (ESVS). Eur J Vasc Endovasc Surg 2018;55:305-68.

18. Varga ZA, Locke-Edmunds JC, Baird RN. A multicenter study of popliteal aneurysms. Joint Vascular Research Group. J Vasc Surg 1994;20:171-7.

19. Carpenter JP, Barker CF, Roberts B, et al. Popliteal artery aneurysms: current management and outcome. J Vasc Surg 1994;19:65-72; discussion 72-3.

20. Huang Y, Gloviczki P, Noel AA, et al. Early complications and long-term outcome after open surgical treatment of popliteal artery aneurysms: is exclusion with saphenous vein bypass still the gold standard? J Vasc Surg 
2007;45:706-713; discussion 713-5.

21. Antonello M, Frigatti P, Battocchio P, et al. Endovascular treatment of asymptomatic popliteal aneurysms: 8-year concurrent comparison with open repair. J Cardiovasc Surg (Torino) 2007;48:267-74.

22. Antonello M, Frigatti P, Battocchio P, et al. Open repair versus endovascular treatment for asymptomatic popliteal artery aneurysm: results of a prospective randomized study. J Vasc Surg 2005;42:185-93.

23. Curi MA, Geraghty PJ, Merino OA, et al. Mid-term outcomes of endovascular popliteal artery aneurysm repair. J Vasc Surg 2007;45:505-10.

24. Pulli R, Dorigo W, Fargion A, et al. Comparison of early and midterm results of open and endovascular treatment of popliteal artery aneurysms. Ann Vasc Surg 2012;26:809-18.

25. Coelho A, Meira J. "Type II Endoleak" and Popliteal Artery Aneurysm Rupture after Surgical Repair. Eur J Vasc Endovasc Surg 2019;58:256.

26. van Santvoort HC, de Vries JP, van de Mortel R, et al. Rupture of a popliteal artery aneurysm 10 years after surgical repair. Vascular 2006;14:227-30.

27. Tsilimparis N, Dayama A, Ricotta JJ 2nd. Open and endovascular repair of popliteal artery aneurysms: tabular

Cite this article as: Ge J, Wang T, Zhao J, Yuan D, Huang B, Yang Y. Comparison of popliteal artery aneurysm outcomes after open repair and endovascular repair: reducing post-operative type II endoleak and sac enlargement. Ann Transl Med 2021;9(22):1688. doi: 10.21037/atm-21-5631 review of the literature. Ann Vasc Surg 2013;27:259-65.

28. Wang T, Zhao J, Yuan D. Successful surgical management of a ruptured popliteal artery aneurysm with acute common peroneal nerve neuropathy: A rare case. Vascular 2021;29:256-9.

29. Mehta M, Champagne B, Darling RC 3rd, et al. Outcome of popliteal artery aneurysms after exclusion and bypass: significance of residual patent branches mimicking type II endoleaks. J Vasc Surg 2004;40:886-90.

30. Dragas M, Zlatanovic P, Koncar I, et al. Effect of Intraoperative Intra-arterial Thrombolysis on Long Term Clinical Outcomes in Patients with Acute Popliteal Artery Aneurysm Thrombosis. Eur J Vasc Endovasc Surg 2020;59:255-64.

31. Schwarz W, Berkowitz H, Taormina V, et al. The preoperative use of intraarterial thrombolysis for a thrombosed popliteal artery aneurysm. J Cardiovasc Surg (Torino) 1984;25:465-8.

32. Kropman RH, Schrijver AM, Kelder JC, et al. Clinical outcome of acute leg ischaemia due to thrombosed popliteal artery aneurysm: systematic review of 895 cases. Eur J Vasc Endovasc Surg 2010;39:452-7.

(English Language Editor: L. Huleatt) 